\title{
Genistein protects against ultraviolet B- induced wrinkling and photoinflammation in in vitro and in vivo models
}

\author{
Sheau-Chung Tang ${ }^{1} \mathbb{D}$, Yu-Ping Hsiao ${ }^{2,3^{*}}$ and Jiunn-Liang $\mathrm{Ko}^{2,4^{*}}$
}

\begin{abstract}
Background: Chronic exposure to ultraviolet (UV) rays causes severe skin damage by inducing oxidative stress and inflammation. Identifying a safe and natural substance for skin protection is a crucial research goal.

Objective: The aim of this study was to clarify the effects of genistein on skin inflammation and photoaging by using 3 models (humans: skin parameters; animals: wrinkle formation; and cells: anti-inflammatory effects).

Methods: Food frequency questionnaire data and serum and skin parameter data from 120 volunteers (a group with a genistein-rich diet [RG group] and a control group). Human keratinocytes were pretreated with genistein before ultraviolet B (UVB) irradiation. Genistein was topically applied to the dorsal skin of rats.

Results: The blood samples of the RG group had lower serum uric acid levels and blood urea nitrogen levels. The dynamic elasticity level in the RG group was higher than that in the controls. Genistein pretreatment suppressed the expression of proinflammatory cytokines (CXCL1, IL-1, MIF, and PLANH1) and the proteins released by UVBtreated keratinocytes. Topical application of genistein to the dorsal skin of rats reduced the severity of UVB-induced wrinkling. Both intake and topical application of genistein combated UVB-induced inflammation and aging.
\end{abstract}

Conclusions: Genistein could be used as a safe and natural compound for use in novel anti-inflammatory agents for topical application.

Keywords: Genistein, Ultraviolet B, Skin, Inflammation, Photoaging

\section{Highlights:}

- Genistein suppressed UVB-induced proinflammatory cytokines (CXCL1, IL-1, MIF, and PLANH1) gene and protein levels in skin cells.

- Genistein reduced the skin folds and wrinkles induced by UVB in the animal model.

- Positive correlation between the RG diet and skin hydration.

\footnotetext{
*Correspondence: missyuping@gmail.com; jlko@csmu.edu.tw ${ }^{2}$ Institute of Medicine, Chung Shan Medical University, No.110, Sec. 1, Chien-Kuo N. Road, Taichung 402, Taiwan

Full list of author information is available at the end of the article
}

\section{Background}

UVB is a known common environmental carcinogen that causes skin aging through the production of reactive oxygen species (ROS) [1-3]. Latreille et al. revealed that intake of polyunsaturated fatty acids, such as docosahexaenoic acid and eicosapentaenoic acid, had an inverse association with UVB-induced photoaging [4]. Dietary consumption of aloe sterols was reported to protect mouse skin against chronic UVB damage [5]. These results indicate that foods can prevent skin aging.

The isoflavone genistein (5,7,40-trihydroxyisoflavone) is abundant in vegetable protein sources, such as soybean. Epidemiological and experimental studies have indicated that genistein may prevent certain symptoms and conditions, such as menopausal symptoms, skin

(c) The Author(s). 2022 Open Access This article is licensed under a Creative Commons Attribution 4.0 International License, which permits use, sharing, adaptation, distribution and reproduction in any medium or format, as long as you give

appropriate credit to the original author(s) and the source, provide a link to the Creative Commons licence, and indicate if changes were made. The images or other third party material in this article are included in the article's Creative Commons licence, unless indicated otherwise in a credit line to the material. If material is not included in the article's Creative Commons licence and your intended use is not permitted by statutory regulation or exceeds the permitted use, you will need to obtain permission directly from the copyright holder. To view a copy of this licence, visit http://creativecommons.org/licenses/by/4.0/. 
cancer, heart disease, and melanoma [6-8]. Genistein decreased serum alanine transaminase and aspartate transaminase levels in a model of rat liver cancer [9]. Furthermore, it blocked apoptotic and necrotic pathways by modulating the expression of proapoptotic genes and proteins [10]. Evidently, genistein has multiple biological functions.

Certain foods and beverages, such as green tea, fish oil, and soybean, possess anti-inflammatory and antioxidative properties, which can help prevent or alleviate various conditions, such as hypertension, gout, and allergies [11-13]. Intake of plant protein is a very healthy way of eating. A diet rich in genistein (RG diet) protects against lower-abdominal obesity, inflammation, and insulin resistance [14]. However, whether genistein-rich food additionally affects physical appearance or can prevent skin disease is unknown.

No evidence is available on the anti-inflammatory and antiphotoaging effects of long-term genistein supplementation on UVB-treated human skin models. Therefore, we hypothesized that exposure to genistein would protect against UVB-induced wrinkle formation and photoinflammation in in vitro and in vivo models. In this study, we sought to understand (1) the differences between the physiological activity of human skin in the RG group and the control group, (2) the action of genistein against cell inflammation caused by UVB light exposure, and (3) how genistein can be applied to induce antiwrinkle effects in animals. We administered the food frequency questionnaire (FFQ) to 120 healthy volunteers and analyzed their genistein intake data, serum, whole blood, and urine samples. A cytokine array and animal model were used to determine the mechanism of genistein's protective effects against UVB-induced photoinflammation.

\section{Results}

\section{Genistein reduces blood urea nitrogen level}

The age range of the 120 participants was $54-56$ years. In the RG $(n=56)$ and control groups $(n=64), 68 \%$ and $56 \%$ of the participants were women, respectively. On average, the participants in the RG group had a larger waist than those in the control group, but age and body mass index (BMI) did not differ significantly between the 2 groups (Table 1). No significant intergroup differences were noted in terms of blood electrolytes, such as iron, potassium, sodium, calcium, and chloride (Table 2).

The baseline characteristics of the 2 groups were determined through blood biochemical tests, and a sexbased division was applied (male: blue dots, female: red dots) (Fig. 1). Compared with the control group, the RG group exhibited lower serum uric acid levels $(5.34 \pm 1.13$ $\mathrm{mg} / \mathrm{dL}$ vs. $4.75 \pm 1.21 \mathrm{mg} / \mathrm{dL} ; p=0.023$ )(Fig. 1D).
Table 1 Participants' basic demographic characteristics

\begin{tabular}{lll}
\hline Groups & Control $(\boldsymbol{N}=\mathbf{6 4})$ & $\mathbf{R G}(\boldsymbol{N}=\mathbf{5 6})$ \\
\hline Mean age in years $( \pm$ SD) & $54.04( \pm 12.30)$ & $56.35( \pm 11.55)$ \\
Female & $56 \%$ & $67 \%$ \\
Average waist (inches) & $29.3(23.2 \sim 37.8)$ & $33.08(24.5 \sim 42.0)$ \\
BMI ( \pm SD) & $24.06( \pm 3.75)$ & $23.32( \pm 2.57)$ \\
Duration of RG (months) & - & $167.92( \pm 102)$ \\
\hline
\end{tabular}

All participants had direct sunlight exposure of less than 1 hour per day. The RG group had more than 4 servings/day of plant protein (the control group had 3 or fewer servings). The FFQ categories are described in the "Methods" section

Moreover, triglyceride (TG) levels were significantly higher in the RG group compared with the control group $(160.89 \pm 50 \mathrm{mg} / \mathrm{dL}$ vs $114.84 \pm 44.02 \mathrm{mg} / \mathrm{dL} ; p=$ 0.014) (Fig. 1B). Other lipid metabolism-based parameters, such as low-density lipoprotein (LDL) and highdensity lipoprotein (HDL) levels, were not significantly different between the 2 groups (Fig. 1C). The total ironbinding capacity and free iron concentration, which are correlated with iron storage and transport, were also not significantly different between the 2 groups (Fig. 1E). These results indicate that most of the serum components were evenly distributed, but the RG group exhibited significantly higher TG levels and significantly lower levels of blood urea nitrogen.

\section{Genistein affects skin $\mathrm{pH}$ and pigmentation in vivo}

To analyze the effect of genistein on human skin, different methods were used to analyze the various skin parameters (moisture, elasticity, and pigmentation) of the 2 groups (Table 3). We observed no significant differences between the 2 groups in terms of skin hydration, elasticity (skin extensibility), melanin, or the erythema index. Skin $\mathrm{pH}$ levels were significantly higher in the RG group than in the control group (5.51 \pm 0.79 vs. $5.20 \pm 0.67 ; p$ $=0.05$ ). We further explored whether sex was an influencing factor. We grouped the individuals on the basis of sex and genistein intake and then analyzed the

Table 2 Participants' blood biochemistry data

\begin{tabular}{llll}
\hline Groups & Control $(\boldsymbol{N}=\mathbf{6 4})$ & RG $(\boldsymbol{N}=\mathbf{5 6})$ & $P$-value \\
\hline TIBC & $339.97( \pm 47.63)$ & $337.3( \pm 45.40)$ & 0.69 \\
Fe & $88.16( \pm 37.57)$ & $78.17( \pm 32.46)$ & 0.20 \\
$\mathbf{K}$ & $4.30( \pm 0.35)$ & $4.25( \pm 0.39)$ & 0.54 \\
$\mathrm{Na}$ & $141.5( \pm 1.78)$ & $141.38( \pm 1.73)$ & 0.77 \\
$\mathrm{Cl}$ & $105.07( \pm 1.98)$ & $106.26( \pm 2.35)$ & 0.06 \\
$\mathrm{Ca}$ & $2.26( \pm 0.11)$ & $2.22( \pm 0.15)$ & 0.18
\end{tabular}

The unit of total iron-binding capacity and $\mathrm{Fe}$ is $\mu \mathrm{g} / \mathrm{dL}$. The unit of $\mathrm{K}, \mathrm{Na}, \mathrm{Cl}$, and $\mathrm{Ca}$ levels is $\mathrm{mmol} / \mathrm{L}$. The RG group had more than 4 servings/day of plant protein (the control group had 3 or fewer servings). The FFQ categories are described in the "Methods" section. All values are represented as the mean \pm SD. All data were analyzed using SPSS 18.0. Statistical analysis was performed using one-way ANOVA. A $p$ value less than 0.05 was considered statistically significant. ${ }^{*} p<0.05,{ }^{* *} p<0.01$ 
(A)

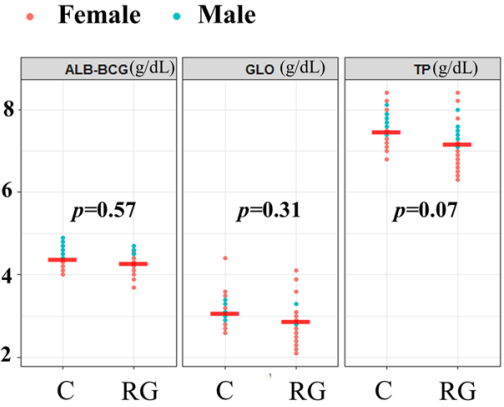

(C)

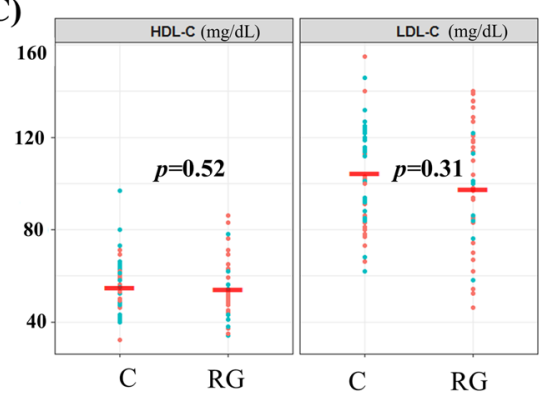

(E)

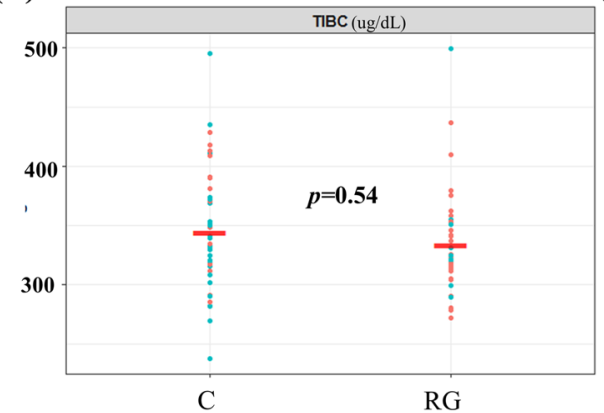

(B)

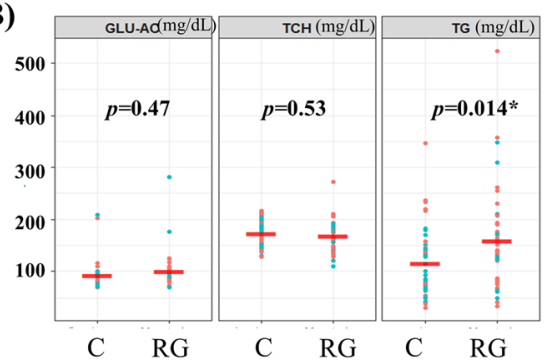

(D)

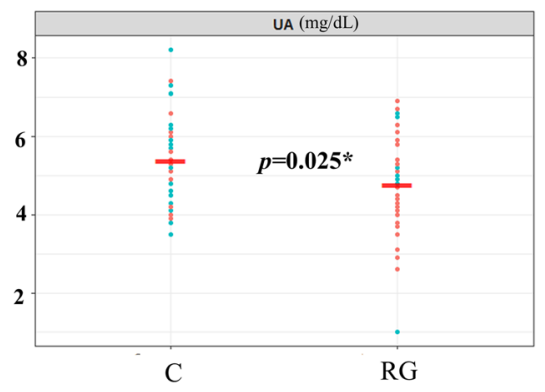

(F)

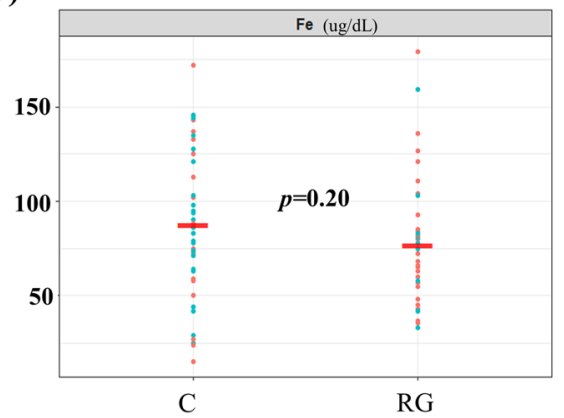

Fig. 1 Biochemical characteristics of the blood samples of 120 participants. Volunteers were divided into the RG group and control (C) group. All values are presented as mean \pm standard deviation (SD). The parametric $t$ test and nonparametric Mann-Whitney $U$ test for independent samples were used to compare groups. A $p$ value of less than 0.05 was considered to indicate statistical significance. ${ }^{*} p<0.05,{ }^{* *} p<0.01$. ALB: albumin; BCG: bromocresol green method, GLO: globulin, TP: total protein, GLU-AC: glucose-AC, TCH: total cholesterol, HDL-C: high-density lipoprotein cholesterol, LDL-C: low-density lipoprotein cholesterol, BUN (blood urea nitrogen). Red dots: female participants, green dots: male participants

Table 3 Participants' skin parameters

\begin{tabular}{llll}
\hline Groups & Control & RG & $P$-value \\
\hline Melanin index & $146.58( \pm 37.11)$ & $140.21( \pm 30.15)$ & 0.40 \\
Erythema index & $157.68( \pm 44)$ & $147.86( \pm 32.15)$ & 0.26 \\
Hydration & $28.18( \pm 6.27)$ & $29.91( \pm 6.14)$ & 0.21 \\
Elasticity & $1752.2( \pm 367.6)$ & $2122.9( \pm 1245.8)$ & 0.40 \\
$\mathrm{pH}$ & $5.20( \pm 0.67)$ & $5.51( \pm 0.79)$ & $0.05^{*}$ \\
\hline
\end{tabular}

The elasticity parameter indicates skin extensibility. Skin parameters were assessed using the Corneometer CM580. The RG group had more than 4 servings/day of plant protein (the control group had 3 or fewer servings). The FFQ categories are described in the "Methods" section. All values are presented as the mean \pm SD. Statistical analysis was performed using one-way ANOVA. A $p$ value less than 0.05 was considered statistically significant. ${ }^{*} p<$ $0.05,{ }^{* *} p<0.01$ aforementioned skin parameters (Fig. 2A). In the RG group, men had a significantly higher erythema index than women $(p=0.04)$. Skin $\mathrm{pH}$ levels were higher among the women than among the men in the RG group. Conversely, skin $\mathrm{pH}$ levels were significantly higher among the men than among the women in the control group $(p=0.02)$.

To understand whether genistein intake had any effect on the various skin parameters, advanced skin elasticity measurement was used to analyze the skin after repeated suction and release. Dynamic elasticity was higher in the RG group than in the control group, and women had significantly higher levels than men in the RG group (Fig. 2B, $p=0.01$ ). These results indicate that compared with the RG group, the control group had lower skin $\mathrm{pH}$ and a higher erythema index. In addition, the RG group, 


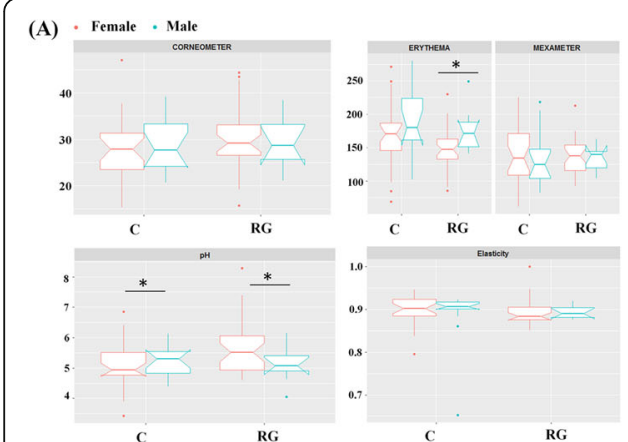

(B)

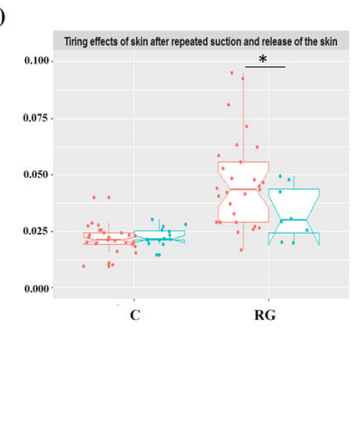

(C)

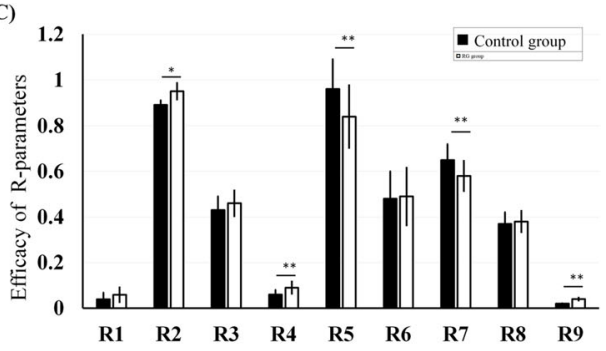

Fig. 2 Participants' skin parameters. (A) Skin hydration was assessed using a corneometer. Basic values for erythema and melanin were measured using a pigmentation probe (mexameter). A skin elastic meter was used to detect total skin elastic. All values are presented as mean \pm SD. Participants were divided into the RG group and control (C) group. Red box: female participants, green box: male participants. All data were analyzed using SPSS version 18.0. A $p$ value of less than 0.05 was considered to indicate statistical significance. ${ }^{*} p<0.05$, ${ }^{* *} p<0.01$. (B) Advanced skin elasticity assay. Skin was subjected to constant negative pressure of $350 \mathrm{mbar}$ in the time-strain mode for $18 \mathrm{~s}$ followed by relaxation for $2 \mathrm{~s}$. (C) Elasticity parameters (R0-R9 and F0-F1) were calculated from the deformation curve and in accordance with the suction applied using the Cutometer MPA580. Bars represent the mean $\pm \mathrm{SD}$. ( $N=5$; each test was performed at least 5 times). R1 indicates skin total deformation; R2, skin gross elasticity; R3, skin amplitude; R4, last minute amplitude; R5, pure elasticity of the skin; R6, skin sagginess; R7, biological elasticity; R8, skin pliability; and R9, skin tiring effect. The black column represents the control group, and the white column represents the RG group. ${ }^{*} p<0.05$; ${ }^{* *} p$ $<0.01$, compared with other groups

especially women in this group, had superior skin elasticity to the control group.

\section{Genistein improves skin elasticity in vivo}

We analyzed a single skin parameter to explore the effect of an RG diet on skin elastin. The $R$ values represent the results at the end of different measurement cycles and are presented in Fig. 2 C. Compared with the control group, the RG group had more pronounced improvements in $\mathrm{R} 2(p=0.04), \mathrm{R} 4, \mathrm{R} 5, \mathrm{R} 7$, and R9 values ( $p<$
0.01). However, no significant differences were observed in R1, R6, and R8 between the 2 groups. Overall, we revealed that skin elasticity significantly improved in the RG group.

A magnification device designed for observing human skin was used to observe the texture of the skin and the distance between the highest peak and lowest valley on the skin's surface. The gray level (GL) was obtained (Fig. $3 \mathrm{~A}$ and $\mathrm{B}$ ) and used to determine skin smoothness, roughness, and wrinkling. The abdomen and arm skin of

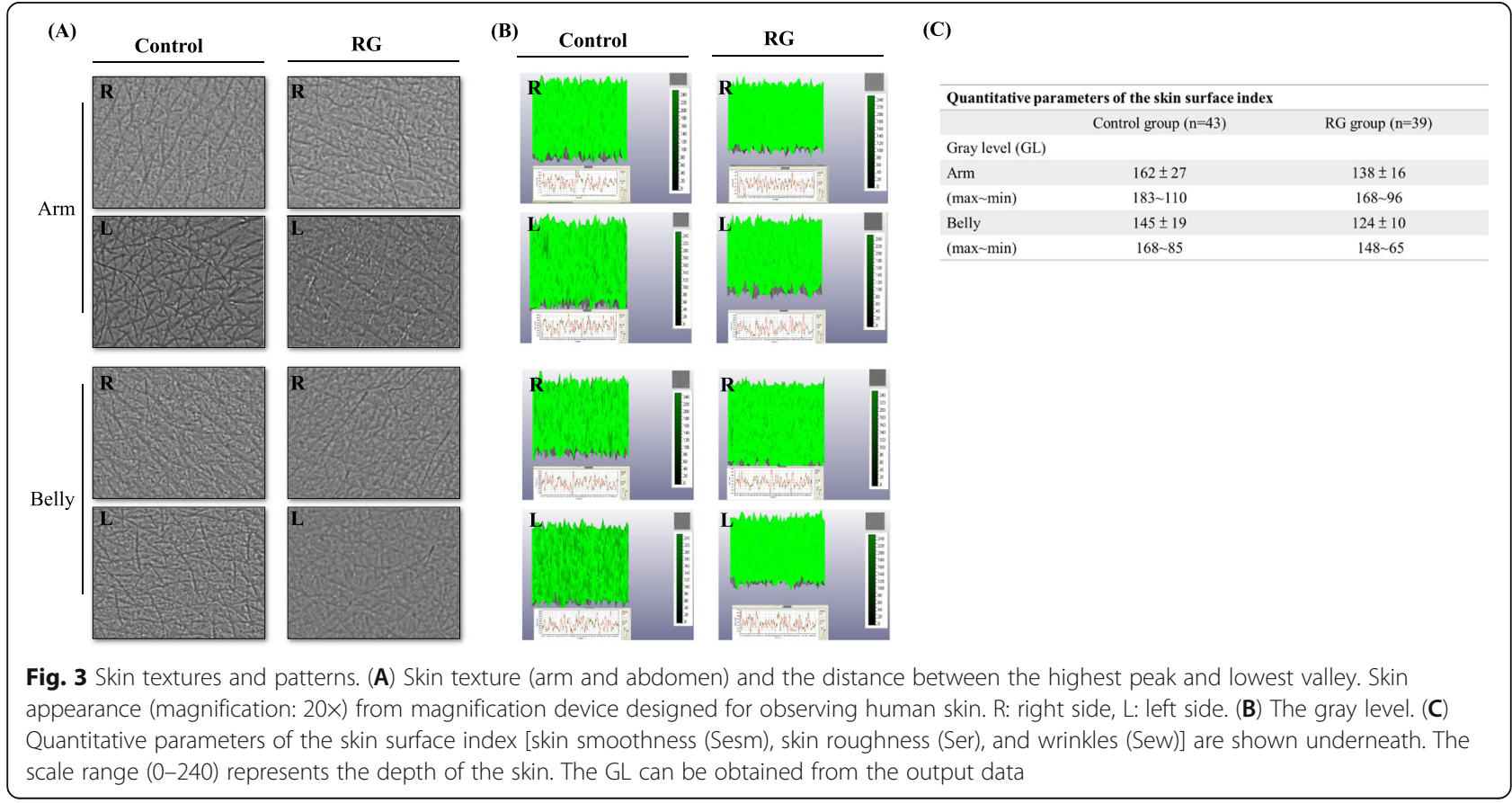


the control group were thicker and had a denser population of skin cells compared with those of the RG group (arm: $162 \pm 27$ vs $138 \pm 16, p=0.053$; abdomen: $145 \pm$ 19 vs $124 \pm 10, p=0.06)$. The GL was obtained from detector the output data, as illustrated in Fig. 3C. The results confirmed that an RG diet could affect skin parameters; such a diet improved skin elasticity, reduced pigmentation, and maintained weak acidity, which protects the skin.

\section{Correlation between genistein intake and skin parameters in vivo}

To understand the correlation between skin parameters and RG diet duration, we analyzed the RG diet duration (in months), pigmentation (melanin and erythema), skin hydration, and skin elasticity in the RG group and the control group. No skin parameter (melanin index, erythema index, or hydration) had a significant correlation with RG diet duration ( $p=0.932,0.446$, and 0.077 , respectively). When only the data of women in the RG group were analyzed, a positive correlation was discovered between skin hydration and RG diet duration $(p=$ 0.044). Moreover, women in the RG group had comparison favorable skin hydration. The RG diet lasted 1 month, and the skin's water retention was $0.014 \%$ higher after this diet $(\beta=0.014 \pm 0.006, p=0.044$; Table 4$)$. Individual analyses of the correlations between skin elasticity parameters and diet duration revealed that R5, R7, and R9 had significant positive correlations with RG diet duration. Using these results and the FFQ scores, we observed that upon the intake of protein derived from soybean, the participants had improved skin hydration. We divided the RG group into 3 groups: those with high,

Table 4 Relationship between skin parameters and time

\begin{tabular}{llll}
\hline Characteristics & \multicolumn{2}{l}{ Time (months) } & \\
\cline { 2 - 4 } & $\boldsymbol{\beta}$ & SEM & $\boldsymbol{P}$-value \\
\hline Melanin index & -0.003 & 0.034 & 0.932 \\
Erythema index & -0.03 & 0.039 & 0.446 \\
Hydration & 0.011 & 0.006 & 0.077 \\
Female & $\mathbf{0 . 0 1 4}$ & $\mathbf{0 . 0 0 6}$ & $\mathbf{0 . 0 4 4 ^ { * }}$ \\
Elasticity & & & \\
R2 & 0.741 & 0.690 & 0.574 \\
R5 & 0.532 & 0.015 & $0.046^{*}$ \\
R7 & 0.221 & 0.030 & $0.022^{*}$ \\
R8 & 0.101 & 0.012 & $0.032^{*}$ \\
\hline
\end{tabular}

Samples were analyzed to determine the relationships between rich genistein intake duration and skin parameters. Linear regression analysis was applied to investigate the relationship between the continuous variables and independent variable. A $p$ value of less than 0.01 was considered statistically significant and labeled ${ }^{* *} . \beta$ : as the duration of a diet rich in genistein diet increased, changes were observed in this parameter. SEM, standard error of the mean. The skin parameters (namely melanin, erythema, hydration, and elasticity) were assessed using the Corneometer CM580
Table 5 Correlation between participants' skin parameters and the FFQ results

\begin{tabular}{lllllll}
\hline $\begin{array}{l}\text { Skin } \\
\text { hydration }\end{array}$ & \multicolumn{7}{l}{ RG diet time (months) } \\
\cline { 2 - 7 } & Ave & SD & $\beta$ & SEM & $n$ & $P$-value \\
\hline Soybean product & & & & & \\
Frequency uptake & & & & & \\
1. High & 34.13 & 7.22 & 7.28 & 2.61 & 16 & $0.007^{* *}$ \\
2. Moderate & 27.97 & 5.56 & 1.12 & 2.31 & 55 & \\
3. Rare & 26.85 & 2.12 & 0.01 & & 7 & \\
\hline
\end{tabular}

FFQ categories are described in the "Methods" section. Subjects who ate more than 4 servings/day were defined as having high intake; those who ate 2-3 servings/day were defined as having moderate intake; and those who ate fewer than 2 servings/day were defined as having low intake. ANOVA (analysis of variance) was used for statistical analysis. Ave, average, $S D$, standard deviation, SEM, standard error of the mean, $n$, number. A $p$ value less than 0.01 was considered statistically significant and labeled **

moderate, and low soybean intake (Table 5). The highintake group had the best skin hydration (7.28 vs. 1.12 [moderate] and 0.01 [low], $p=0.007$ ). These data indicate a positive correlation between RG diet duration, soybean intake, and skin hydration.

\section{Genistein reduces skin cell inflammation caused by UVB irradiation in human keratinocytes}

To demonstrate that genistein reduced the degree of UVB-induced skin aging, human keratinocyte ( $\mathrm{HaCaT})$ cells were pretreated with genistein and then exposed to UVB irradiation. We confirmed the toxicity of genistein for $\mathrm{HaCaT}$ cells by performing an MTT assay, in which $5 \mu \mathrm{M}$ was the treatment concentration selected (Supplementary Fig. 1). We analyzed the expression of proinflammatory cytokines by using a cytokine array; genistein pretreatment reduced the amounts of proinflammatory cytokines (CXCL1, IL-1, and MIF) released by UVB-treated keratinocytes (Fig. 4A). An analysis of pixel density from the aforementioned array was conducted, and of the analyzed proinflammatory cytokines, MIF expression was the most significantly decreased by genistein treatment (Fig. 4B, $p=0.015$ ). To confirm the results of this study, an enzyme-linked immunosorbent assay (ELISA) was used to reveal the performance of genistein in suppressing cytokine release (Fig. 4 C). The cytokine release results for IL-1 and MIF were consistent with those from the cytokine array. The gene expression levels of these cytokines were also analyzed using quantitative polymerase chain reaction (PCR), and the same trend was discovered (Fig. 4 D). The levels of cytokines secreted in response to the UVB-irradiated culture medium were higher than those observed in the control medium. Similarly, genistein treatment released significantly lowered the levels of CXCL1, IL-1, and MIF compared with the UVB irradiation group $(p<0.05)$. These results suggest that decreases in IL-1 and MIF cytokine secretion were significantly genistein dependent. 

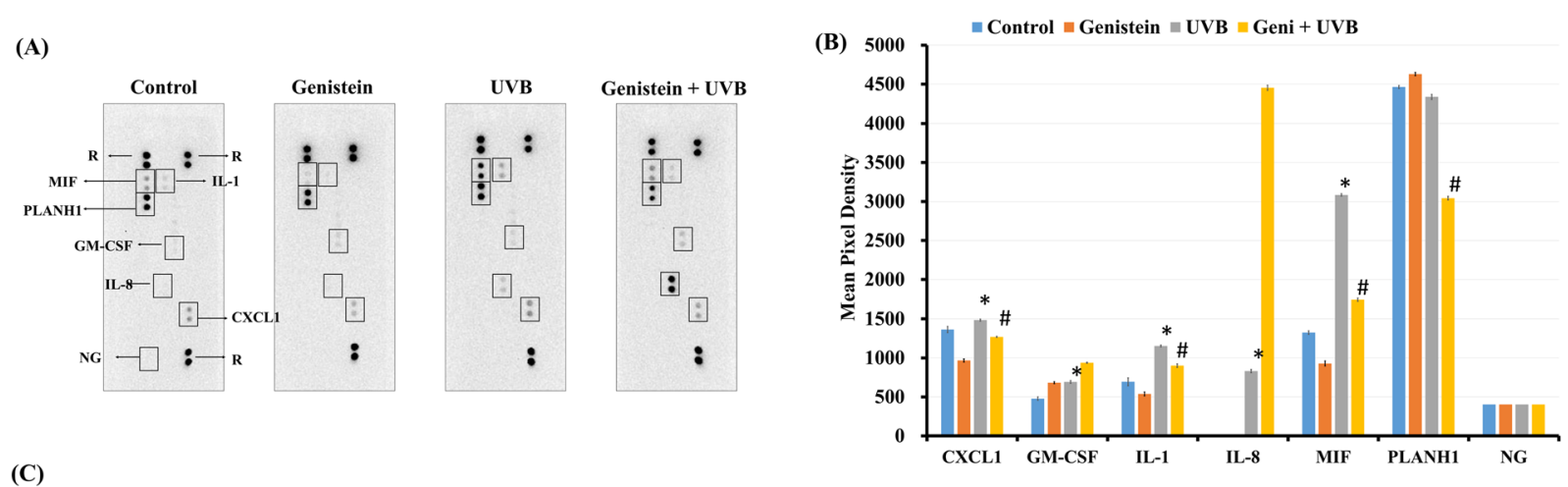

(C)
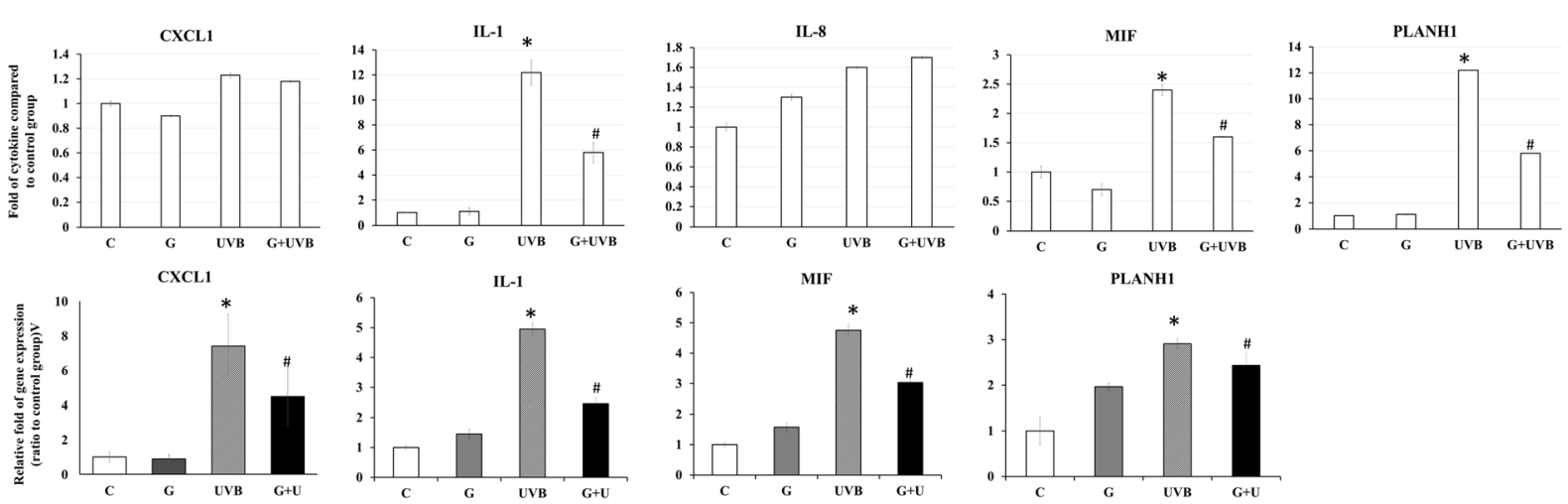

Fig. 4 Effect of genistein on UVB-irradiated skin cells. (A) Cytokine array measurements were performed on cell culture supernatants harvested from cultures pretreated with genistein $(5 \mu \mathrm{M})$ for $24 \mathrm{~h}$ with or without UVB $\left(50 \mathrm{~mJ} / \mathrm{cm}^{2}\right)$ irradiation. Each blot represents the immunoreactive staining of respective antibodies. Staining is absent in the negative control and blank slots. R1, R2, and R3 were used to determine the accuracy of the procedure. NG, negative control group. (B) Cytokine levels were quantified and analyzed using image analysis software. The relative expression levels of cytokines were determined through a comparison of the pixel intensity of the respective blots relative to that of the positive control in the same array. The parametric $t$ test for independent samples was used to determine differences between groups. Values are presented as the mean \pm SD of 2 independent measurements. (C) Commercially available ELISA kits for analyzing the levels of CXCL1, IL-1, MIF, $I L-8$, and PLANH1 were used in accordance with manufacturer instructions to quantify fold changes. Relative cytokine release is expressed as a percentage compared with an untreated control. (D) The relative gene expression levels of cytokines were measured using Q-PCR. ${ }^{*} p<0.05$; ${ }^{* *} p$ $<0.01$, compared with other groups. ${ }^{*} p 0.05$ compared with the UVB group

Topical application of genistein for reducing photoaging in an animal model

An animal model was used to determine whether the topical application of genistein affected UVB-induced skin aging. Two areas and duplicate areas on the dorsal skin of the same rats were marked and treated with $0.1 \%$ or $1 \%$ genistein. The genistein solution was applied to the dorsal skin of the rat for $24 \mathrm{~h}$; the skin of the rats was then exposed to UVB light. The skin of the control and genistein groups $(0.1 \%$ and $1 \%)$ did not change; however, the dorsal skin of the UVB-treated control group had prominent folds and wrinkles (black arrow), with tissue damage noted (Fig. 5).

\section{Discussion}

This study examined the effects of genistein on UVBinduced skin aging and inflammation by using 3 models (in vivo, cell, and animal models). Our results demonstrated that genistein promoted skin hydration, decreased the amounts of UVB-induced proinflammatory cytokines released, and reduced the severity of UVBinduced wrinkles. We suggest that exposure to genistein may be beneficial for preventing light-induced skin aging.

In the cell model, among the 35 proinflammatory cytokines induced by UVB irradiation (Fig. 4), genistein significantly decreased the expression of CXCL1, IL-1, and MIF $(p<0.05)$; among them, genistein had the most significant inhibitory effect on MIF. MIF plays a critical role in the pathogenesis of UVB-induced nonmelanoma skin cancer [15]. MIF overexpression has been implicated in chronic inflammatory diseases and malignancies [16]. MIF deficiency significantly reduces acute inflammatory responses in the skin following UVB exposure [17]. We demonstrated that genistein effectively inhibited the expression of MIF. However, CXCL1, IL-1, and MIF cytokines did not differ in the blood samples of the two groups totaling 120 healthy participants. We speculate that MIF may be an indicator of skin cancer, meaning that it does not appear in large quantities in the 


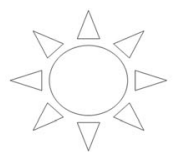

\section{Control}
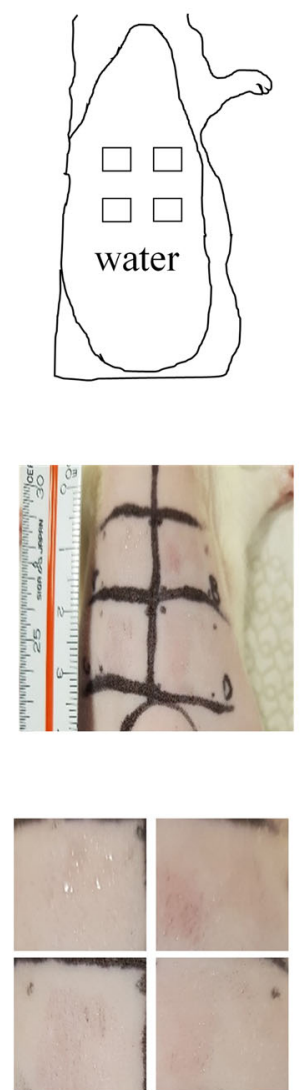

Genistein
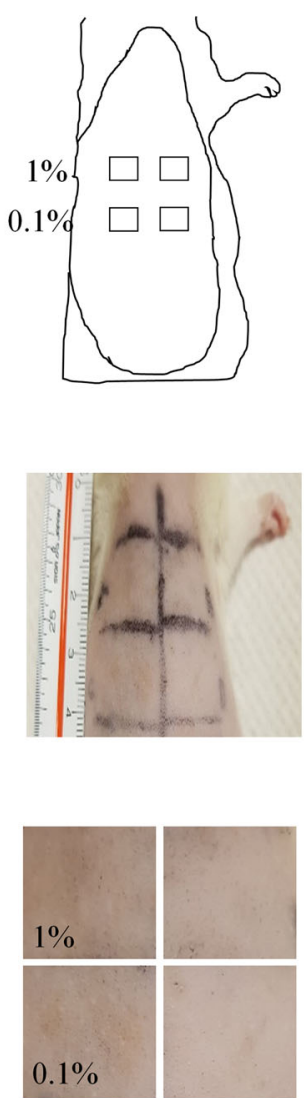

UVB
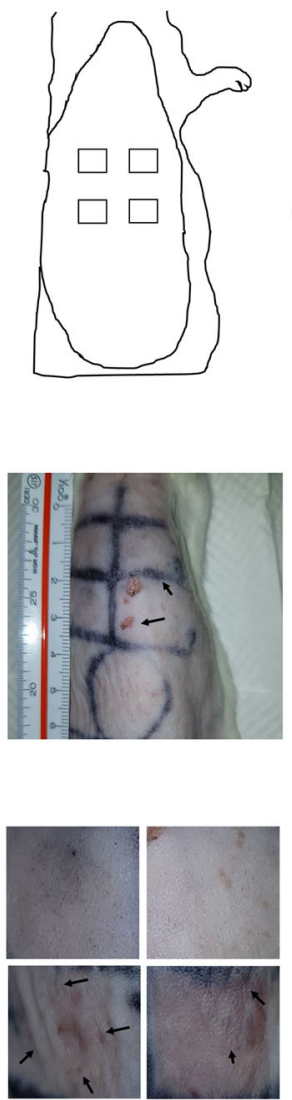

Genistein+UVB
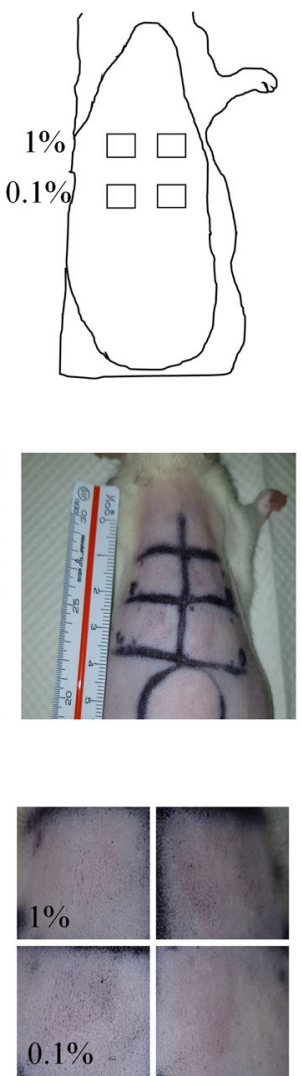

Fig. 5 Animal model observations from the application of genistein. Male SD rats (3 to 4 weeks old) were divided into 4 groups ( $n=6$ per group). The hair on the back of the rat was removed 2 to 3 days before the experiments. The back of each animal was marked with India ink, and the marked area was divided into 4 squares $\left(0.3 \times 0.3 \mathrm{~cm}^{2}\right.$ each); these squares were treated with $10 \mu \mathrm{L}$ of each concentration $(0.1 \%$ and $1 \%)$ of genistein solution in water, and one square was treated with water as a control area. The rats were pretreated with genistein once before they were exposed to UVB radiation $\left(50 \mathrm{~mJ} / \mathrm{cm}^{2}\right.$ ); subsequently, skin wrinkling was investigated (magnification: $10 \times$ )

blood of healthy people. However, the skin cell line used in this study was an immortalized model. Skin cell lines were well modeled to observe the regulatory influence of genistein on MIF. We suggest that genistein has potential use for skin cancer treatment.

Studies have shown that UVB irradiation causes skin injuries and oxidative damage through various pathways, such as the mitogen-activated protein kinase and nuclear factor-kappa beta $(\mathrm{NF}-\kappa \mathrm{B}) / \mathrm{p} 65$ pathways $[18,19]$. We also further proved that some proinflammatory cytokines (IL-6, IL-8, MCP-1, and COX-2) were stimulated by UVB irradiation through the NF- $\mathrm{kB} / \mathrm{p} 65$ pathway in keratinocytes and a mouse model [1]. However, those cytokines were also analyzed in the cell model of the present study and found to be unaffected by genistein treatment. Therefore, we surmise that the effects of genistein were not related to the NF- $\mathrm{kB} / \mathrm{p} 65$ pathway. Future experiments can explore the pathways through which genistein inhibits MIF expression in UVBirradiated skin cells.

Through animal experiments (Fig. 4) and those involving human participants (Fig. 3), we confirmed that genistein significantly reduced inflammation and the severity of UVB-induced wrinkling. Genistein exhibits beneficial effects against skin aging through the increased synthesis of collagen and hyaluronic acid [20], the reduction of MMP expression, and the stimulation of fibroblast proliferation [21]. Collagen is synthesized primarily by fibroblasts residing within the dermis and is the cause of skin's strength and elasticity, which are closely related to skin hydration and the formation of wrinkles. Our results demonstrated that genistein improved skin 
elasticity, reduced pigmentation, and maintained weak skin acidity. We must clarify the mechanism of genistein's regulation of skin hydration through future investigations.

Studies have indicated that genistein has estrogen-like effects $[22,23]$ and has a higher affinity with estrogen receptors than other isoflavones have [24, 25]. Therefore, genistein's effects are usually more pronounced in women. In our study, women had higher hydration and elasticity levels as well as lower LDL levels than the mean levels in the control and treatment groups (Fig. 1). Sex and hormones may explain these findings. However, the age range of the 120 participants was $54-56$ years. In the RG group, the proportion of menopausal women was $44.7 \%$ (17 out of 38 women); in the control group, the proportion was $45.7 \%$ ( 16 out of 35 women). This result indicates that gender and menopausal status are just one of many influencing factors. One study indicated that treating postmenopausal skin with estrogen achieved superior results when isoflavones were used [22]. This result is not consistent with our results; more female participants are required to confirm whether hormones affect skin parameters.

Genistein has been tested in cosmetic antiaging preparations, and it has achieved notable results in terms of skin elasticity improvement, antiphotoaging, and skin cancer prevention. Cosmetic creams containing genistein have been used to alleviate skin dryness and wrinkles [26]. However, few studies have explored the effects of long-term genistein application on the physiological condition or $\mathrm{pH}$ of the skin. The RG diet duration (the time required for a genistein-rich diet to improve health) in the present study was long (average: $168 \pm 102$ months); thus, our data can be used to observe the relationship between genistein intake and duration. Our data demonstrated that the skin $\mathrm{pH}$ levels of all participants were within the normal range, although they were higher in the RG group than in the control group. One study demonstrated that women have lower skin $\mathrm{pH}$ than men [27]. However, we posit that genistein intake can reduce the quantity of acid deposits on the skin and higher the skin's pH. Animal protein is metabolized into lactic acid and secreted by the skin and through sweat. A study indicated that nutrient metabolism may affect the skin through the microbiota [28]. Dietary intake of fiber and polysaccharides can reduce the accumulation of acidic substances through changes to probiotics in the gastrointestinal tract, reducing the severity of skin acne and hair follicle inflammation [29]. Whether an RG diet regulates lactobacillus and then affects the skin's $\mathrm{pH}$ is of interest. Many studies assessing nutrients and skin inflammation have been performed using an atopic dermatitis model [30, 31]. Few related studies have used healthy human skin because many variables can affect the results. In this study, we used various methods to demonstrate that high genistein intake was beneficial in both in vivo and in vitro models.

\section{Conclusion}

Genistein provided protection against UVB-induced inflammation. We demonstrated that an RG diet could affect the skin condition of participants; their skin exhibited favorable elasticity and hydration. Genistein suppressed the UVB-induced expression of proinflammatory cytokines (CXCL1, IL-1, and MIF) in skin keratinocytes. Topically applied genistein effectively reduced the appearance of wrinkles caused by sun exposure in an animal model. The experimental process and results are presented in a graphical abstract. Genistein is a safe and natural compound that could be employed within novel anti-inflammatory agents for topical application.

\section{Methods \\ Reagents and chemicals}

Genistein and phosphate-buffered saline were purchased from Sigma-Aldrich (St. Louis, MO, USA). Dulbecco's modified Eagle's medium (DMEM), penicillin/streptomycin, and fetal bovine serum were purchased from Gibco-Invitrogen (Carlsbad, CA, USA). The Cutometer MPA580 (C+K, Köln, Germany) skin detector was provided by the Department of Dermatology of Hualien Tzu Chi Hospital (Buddhist Tzu Chi Medical Foundation, Taiwan). ELISA kits for CXCL1 (cat. DGR00B), IL$1 \beta$ (cat. DLB50), MIF (cat. SMF00B), and Serpin E1/ PAI-1 (cat. DSE100) were obtained from R\&D Systems, Inc. (Minneapolis, MN, USA). The Human IL-8/CXCL8 Quantikine ELISA Kit (S8000C) was obtained from BD OptEIA Set and used in accordance with the manufacturer's instructions (Becton Dickinson, NY, USA).

\section{Study design and participants}

A total of 137 participants (17 with incomplete information) were recruited between August 2016 and January 2018 for a 2-year study conducted by the Department of Dermatology, Hualien Tzu Chi Hospital, Taiwan. Informed consent was obtained from all participants, and institutional review board (IRB) approval was obtained prior to study commencement (IRB 104-44-A, Department of Dermatology, Hualien Tzu Chi Hospital, Buddhist Tzu Chi Medical Foundation). All participants were assessed using the FFQ [32-35]. The widely used instrument has correlation coefficients between 0.29 and 0.47 [36]. The inclusion criteria were as follows: healthy individuals aged 31-80 years (to confirm the effect of a long-term RG diet on the skin, the participants had to be older than 30 years) with $\mathrm{BMI}<35 \mathrm{~kg} / \mathrm{m}^{2}$. The exclusion criteria were as follows: history of skin cancer, 
photosensitivity disorder or atopy, sunbathing history or use of sunbeds in the preceding 3 months, photoactive medication administration, pregnancy, smoking status, alcohol use disorder, and substance use disorder. Relevant information-average daily exposure to strong sunlight, alcohol consumption habits (e.g., frequency, type, and quantity of alcoholic drinks consumed), dietary intake assessment results, and supplementary intake (vitamin pills, etc.) - was acquired. The FFQ covered 23 food items, and the frequency categories were 4-6 servings/ day, less than 2 servings/day, 1-3 servings/week, and almost never [14, 36]. The RG group had more than 4 servings/day of plant protein, the control group had 3 or fewer servings. To analyze the relationship between genistein exposure and skin parameters, those in the RG group were divided into 3 subgroups on the basis of their soybean intake (high group: more than 4 servings/ day; moderate group: 2-3 servings/day; low group: less than 2 servings/day). The volunteers were asked to fast for $8 \mathrm{~h}$ before blood samples were drawn for biochemistry measurements. Analyses of TG, HDL, LDL, fasting glucose, and creatinine levels as well as general biochemical analyses were performed at the Department of Laboratory Medicine, Hualien Tzu Chi Hospital, Taiwan. Plasma, serum, and urine samples were taken, and skin images were captured. The morphological characterization of skin samples was conducted after optimized images were captured using a digital microscope (Leica ATC 2000) fitted with a camera (40× resolution).

\section{Skin parameter assay}

Skin hydration was assessed using the Corneometer CM580. The detectors of MPA580 included a corneometer, $\mathrm{pH}$ meter, mexameter, and elasticity probes. Additional parameters that could be assessed using the skin elasticity probe included $U$ and $R$ values. A measuring capacitor shows changes in capacitance in accordance with the moisture content of a sample [37]. The mechanical properties of the epidermis samples were determined using a noninvasive suction-based skin elastic meter equipped with a 2-mm measuring probe (Supplementary Fig. 2). Interference from sunlight and cosmetic lotion was avoided. Participants' forearm areas and abdominal area covered by clothing were selected for testing, and $3-\mathrm{cm} \times 3-\mathrm{cm}$ regions were marked on both forearms and the belly (right and left sides). The skin was marked as the basis of the aperture of the probe at a constant negative pressure of $350 \mathrm{mbar}$ in the timestrain mode for $18 \mathrm{~s}$, followed by relaxation for $2 \mathrm{~s}$; the procedure was performed twice. Various $R$ values could be obtained to clarify the operation. We explain these parameters on the basis of two studies in which this technique has been applied [38, 39]. The $\mathrm{R}$ parameters provided by the cutometer are R2 (gross elasticity or overall elasticity of the skin), R5 (net elasticity), R7 (biological elasticity; the ratio of elastic recovery to total deformation), and R8 (pliability; the ability of the skin to return to its original state). The skin deformation curves generated by the cutometer were mathematically and statistically analyzed. Basic values for erythema and melanin were obtained using a pigmentation probe (mexameter). All readings were acquired at $25 \pm 2{ }^{\circ} \mathrm{C}$ and 40 to $50 \%$ relative humidity.

\section{Animal model, cell culture, and drug treatments}

Animals were purchased from the National Laboratory Animal Center and housed at the Laboratory Animal Center, Chung-Shan University. The animal feeding approach was that used in our previous study [1]. We determined the effects of the topical application of aqueous genistein solutions ( 0.1 and $1 \%)$ on skin parameters. The back of each animal was marked with India ink, and the marked area was divided into 4 squares. Each group contained 6 animals. All animal experimental protocols were approved by the Ethics Committee of Chung-Shan Medical University Experimental Animal Center (Institutional Animal Care and Use Committee [IACUC] approval number: 1837). For the cell culture model, $\mathrm{HaCaT}$ cells were grown in DMEM at $37{ }^{\circ} \mathrm{C}$ in a humidified incubator with a $5 \% \mathrm{CO}_{2}$ atmosphere. Cells were seeded in 6-well plates at a density of $2 \times 10^{5}$ cells per well and cultured for $24 \mathrm{~h}$ in DMEM with genistein $(5 \mu \mathrm{M})$. The supernatant was collected and evaluated using a cytokine array.

\section{UVB exposure}

UVB light was supplied using a closely spaced array of KLBiotech STS-1 sunlamps, and the protocol was the same as that in our previous study [1]. The output energy of the UVB (290-320 nm) light was measured using a UVB photometer (LT Lutron, UV-340A photometer, International Light, Taiwan). The energy output was 1.5 $\mathrm{mW} / \mathrm{cm}^{2}$ and corrected using the Dermaray UV meter and detector (Gigahertz-Optik, Pochheim, Germany). All procedures were conducted in accordance with relevant guidelines and regulations for both human and animal studies.

\section{Reverse transcription PCR, quantitative real-time PCR, and cytokine assay}

Total RNA was isolated using Trizol reagent (Life Technologies, Grand Island, NY, USA) in accordance with the manufacturer's instructions. Reverse transcription PCR and quantitative real-time PCR (qRT-PCR were performed as previously described [1]. The primers for Q-PCR amplification are presented in Supplementary Table 1. Cell-conditioned media were centrifuged for 5 min at $1200 \times g$, and supernatants were collected before 
evaluation of cell cytokine secretion profiles. The levels of IL-8 in the cell supernatants were measured using a BD Opt EIA Set Human IL-8 ELISA kit following the manufacturer's instructions (Becton Dickinson). Levels of other cytokines (CXCL1, IL-1, MIF, and PLANH1) were determined using an ELISA kit.

\section{Statistical analysis}

The measured values obtained for various parameters were analyzed using SPSS version 18.0. The parametric $t$ test for independent samples was used to compare groups. Analysis of variance (ANOVA) was used for comparisons of more than 2 groups. A $p$ value of $<0.05$ was considered statistically significant.

\begin{abstract}
Abbreviations
AST: Aspartate transaminase; ALT: Alanine transaminase; BMI: Body mass index; BUN: Blood urea nitrogen; ER alpha: Estrogen receptor alpha; FBS: Fetal bovine serum; FFQ: Food frequency questionnaire; MMP: Metalloproteinase; NSAIDs: Nonsteroidal anti-inflammatory drugs; PUFAs: Polyunsaturated fatty acids; Real-time PCR: Real-time polymerase chain reaction; ROS: Reactive oxygen species; TG: Triglyceride; TIBC: Total iron-binding capacity; UVB: Ultraviolet B
\end{abstract}

\section{Supplementary Information}

The online version contains supplementary material available at https://doi. org/10.1186/s12263-022-00706-x.

Additional file 1: Supplementary Fig. 1. Cell viability following treatment with various concentrations of genistein for the indicated time periods ( 24 and $48 \mathrm{~h}$ ). An MTT assay was used to determine the effect of genistein treatment on $\mathrm{HaCaT}$ cell viability. Data are presented as mean \pm SD from 3 experiments $(n=6)$. ${ }^{*} p<0.05 ;{ }^{* *} p<0.01$.

Additional file 2: Supplementary Fig. 2. Cutometer MPA580 measurements of the elasticity, hydration, pigmentation, and $\mathrm{pH}$ levels of the skin of participants' upper limbs. Skin hydration was assessed using the Corneometer CM580. The detectors of the Corneometer CM580 included a corneometer, $\mathrm{pH}$ meter, mexameter, and elasticity probes. Participants' upper and inner arm areas and abdomen area were selected for testing.

Additional file 3: Supplementary Table 1. Primer sequences for cDNA amplification of selected human genes.

\section{Acknowledgements}

The authors thank all their coworkers who assisted with this study. They also sincerely thank those who participated in this study. Dr. Yang Jen-Hung is thanked for his encouragement related to this study.

\section{Authors' contributions}

Sheau-Chung Tang: writing —-reviewing and editing. Jiunn-Liang Ko: supervision and investigation. Yu-Ping Hsiao: methodology and resources. The authors read and approved the final manuscript.

\section{Funding}

This work was supported by grants from the Ministry of Science and Technology (MOST 108-2314-B-025-002-MY2), (MOST 110-2320-B-025-001-) and Chung Shan Medical University (CSH-2019-D-008) and (CSH-2021-C-028).

\section{Availability of data and materials}

The data sets used or analyzed are available from the corresponding author upon reasonable request.

\section{Declarations}

\section{Ethics approval and consent to participate}

Consent for the use of human material was provided by the Research Ethics Committee, Hualien Tzu Chi Hospital, Buddhist Tzu Chi Medical Foundation (Identifier: IRB 104-44-A). All animal experimental protocols were approved by the IACUC of Chung-Shan Medical University Experimental Animal Center (approval number: 1837).

\section{Consent for publication}

Not applicable.

\section{Competing interests}

The authors have no conflicts of interest to declare.

\section{Author details}

${ }^{1}$ Department of Nursing, National Taichung University of Science and Technology, Taichung 403, Taiwan. ${ }^{2}$ Institute of Medicine, Chung Shan Medical University, No.110, Sec. 1, Chien-Kuo N. Road, Taichung 402, Taiwan. ${ }^{3}$ Department of Dermatology, Chung Shan Medical University Hospital, No.110, Sec. 1, Chien-Kuo N. Road, Taichung 402, Taiwan. ${ }^{4}$ Department of Medical Oncology and Chest Medicine, Chung Shan Medical University Hospital, Taichung 402, Taiwan.

Received: 1 September 2021 Accepted: 8 February 2022

Published online: 24 February 2022

\section{References}

1. Tang SC, Liao PY, Hung SJ, Ge JS, Chen SM, Lai JC, et al. Topical application of glycolic acid suppresses the UVB induced IL-6, IL-8, MCP-1 and COX-2 inflammation by modulating NF-kappaB signaling pathway in keratinocytes and mice skin. J Dermatol Sci. 2017;86(3):238-48. https://doi.org/10.1016/j. jdermsci.2017.03.004

2. Hung SJ, Tang SC, Liao PY, Ge JS, Hsiao YP, Yang JH. Photoprotective potential of glycolic acid by reducing NLRC4 and AIM2 inflammasome complex proteins in UVB radiation-induced normal human epidermal keratinocytes and mice. DNA Cell Biol. 2017;36(2):177-87. https://doi.org/1 $0.1089 /$ dna.2016.3471.

3. Gegotek A, Jarocka-Karpowicz I, Skrzydlewska E. Synergistic cytoprotective effects of rutin and ascorbic acid on the proteomic profile of 3D-cultured keratinocytes exposed to UVA or UVB radiation. Nutrients. 2019;11(11). https://doi.org/10.3390/nu11112672.

4. Latreille J, Kesse-Guyot E, Malvy D, Andreeva V, Galan P, Tschachler E, et al. Association between dietary intake of $n-3$ polyunsaturated fatty acids and severity of skin photoaging in a middle-aged Caucasian population. J Dermatol Sci. 2013;72(3):233-9. https://doi.org/10.1016/j.jdermsci.2013.07.006.

5. Misawa E, Tanaka M, Saito M, Nabeshima K, Yao R, Yamauchi K, et al. Protective effects of Aloe sterols against UVB-induced photoaging in hairless mice. Photodermatol Photoimmunol Photomed. 2017;33(2):101-11. https://doi.org/10.1111/phpp.12286.

6. Messina M, Messina V. Soyfoods, soybean isoflavones, and bone health: a brief overview. J Ren Nutr. 2000;10(2):63-8. https://doi.org/10.1016/S1051-22 76(00)90001-3.

7. Wei $H$, Bowen $R$, Zhang $X$, Lebwohl M. Isoflavone genistein inhibits the initiation and promotion of two-stage skin carcinogenesis in mice. Carcinogenesis. 1998;19(8):1509-14. https://doi.org/10.1093/carcin/19.8.1509.

8. Record IR, Broadbent JL, King RA, Dreosti IE, Head RJ, Tonkin AL. Genistein inhibits growth of B16 melanoma cells in vivo and in vitro and promotes differentiation in vitro. Int J Cancer. 1997;72(5):860-4. https://doi.org/10.1 002/(SICI)1097-0215(19970904)72:5<860::AID-IJC24>3.0.CO;2-B.

9. Ganai AA, Husain M. Genistein attenuates D-GalN induced liver fibrosis/ chronic liver damage in rats by blocking the TGF-beta/Smad signaling pathways. Chem Biol Interact. 2017;261:80-5. https://doi.org/10.1016/j.cbi.2 016.11.022.

10. Ganai AA, Khan AA, Malik ZA, Farooqi H. Genistein modulates the expression of NF-kappaB and MAPK ( $p-38$ and ERK1/2), thereby attenuating $d$ galactosamine induced fulminant hepatic failure in Wistar rats. Toxicol Appl Pharmacol. 2015;283(2):139-46. https:/doi.org/10.1016/j.taap.2015.01.012.

11. Melough MM, Chun OK. Dietary furocoumarins and skin cancer: a review of current biological evidence. Food Chem Toxicol. 2018;122:163-71. https:// doi.org/10.1016/j.fct.2018.10.027. 
12. Clarke KA, Dew TP, Watson REB, Farrar MD, Osman JE, Nicolaou A, et al. Green tea catechins and their metabolites in human skin before and after exposure to ultraviolet radiation. J Nutr Biochem. 2016;27:203-10. https:// doi.org/10.1016/j.jnutbio.2015.09.001.

13. Yum HW, Kim SH, Kang JX, Surh YJ. Amelioration of UVB-induced oxidative stress and inflammation in fat- 1 transgenic mouse skin. Biochem Biophys Res Commun. 2018;502(1):1-8. https://doi.org/10.1016/j.bbrc.2018.05.093.

14. Chuang SY, Chiu THT, Lee CY, Liu TT, Tsao CK, Hsiung CA, et al. Vegetarian diet reduces the risk of hypertension independent of abdominal obesity and inflammation: a prospective study. J Hypertens. 2016;34(11):2164-71. https://doi.org/10.1097/HJH.0000000000001068.

15. Kang TH, Park HM, Kim YB, Kim H, Kim N, Do JH, et al. Effects of red ginseng extract on UVB irradiation-induced skin aging in hairless mice. J Ethnopharmacol. 2009;123(3):446-51. https://doi.org/10.1016/j.jep.2009.03.022.

16. Shimizu T, Abe R, Ohkawara A, Nishihira J. Ultraviolet B radiation upregulates the production of macrophage migration inhibitory factor (MIF) in human epidermal keratinocytes. J Invest Dermatol. 1999;112(2):210-5. https://doi.org/10.1046/j.1523-1747.1999.00486.x.

17. Martin J, Duncan FJ, Keiser T, Shin S, Kusewitt DF, Oberyszyn T, et al. Macrophage migration inhibitory factor (MIF) plays a critical role in pathogenesis of ultraviolet-B (UVB)-induced nonmelanoma skin cancer (NMSC). FASEB J. 2009;23(3):720-30. https://doi.org/10.1096/fj.08-119628.

18. Bosch R, Philips N, Suárez-Pérez J, Juarranz A, Devmurari A, ChalensoukKhaosaat J, et al. Mechanisms of photoaging and cutaneous photocarcinogenesis, and photoprotective strategies with phytochemicals. Antioxidants (Basel). 2015;4(2):248-68. https://doi.org/10.3390/antiox4020248.

19. Kim C, Sano Y, Todorova K, Carlson BA, Arpa L, Celada A, et al. The kinase p38 alpha serves cell type-specific inflammatory functions in skin injury and coordinates pro- and anti-inflammatory gene expression. Nat Immunol. 2008;9(9):1019-27. https://doi.org/10.1038/ni.1640.

20. Sudel KM, et al. Novel aspects of intrinsic and extrinsic aging of human skin: beneficial effects of soy extract. Photochem Photobiol. 2005;81(3):581-7. https://doi.org/10.1562/2004-06-16-RA-202.1.

21. Kim SY, Kim SJ, Lee JY, Kim WG, Park WS, Sim YC, et al. Protective effects of dietary soy isoflavones against UV-induced skin-aging in hairless mouse model. J Am Coll Nutr. 2004;23(2):157-62. https://doi.org/10.1080/0731 5724.2004.10719356.

22. Patriarca MT, Barbosa de Moraes AR, Nader HB, Petri $V$, Martins JRM, Gomes RCT, et al. Hyaluronic acid concentration in postmenopausal facial skin after topical estradiol and genistein treatment: a double-blind, randomized clinical trial of efficacy. Menopause. 2013;20(3):336-41. https://doi.org/10.1 097/gme.0b013e318269898c

23. Xu Y, Li X, Zhang H, Wu Y, Zhang J, Li J, et al. China Tongxinluo Study for myocardial protection in patients with Acute Myocardial Infarction (CTSAMI): rationale and design of a randomized, double-blind, placebocontrolled, multicenter clinical trial. Am Heart J. 2020;227:47-55. https://doi. org/10.1016/j.ahj.2020.06.011.

24. Ewies AA. Phytoestrogens in the management of the menopause: up-todate. Obstet Gynecol Surv. 2002;57(5):306-13. https://doi.org/10.1097/ 00006254-200205000-00023.

25. Chen LR, Ko NY, Chen KH. Isoflavone supplements for menopausal women: a systematic review. Nutrients. 2019;11(11). https://doi.org/10.3390/nu11112649.

26. Ehlers C, Ivens UI, Møller ML, Senderovitz T, Serup J. Comparison of two pH meters used for skin surface $\mathrm{pH}$ measurement: the $\mathrm{pH}$ meter ' $\mathrm{pH} 900$ ' from Courage \& Khazaka versus the $\mathrm{pH}$ meter ' 1140 ' from Mettler Toledo. Skin Res Technol. 2001;7(2):84-9. https://doi.org/10.1034/j.1600-0846.2001.70205.x.

27. Ehlers C, Ivens UI, Møller ML, Senderovitz T, Serup J. Females have lower skin surface $\mathrm{pH}$ than men. A study on the surface of gender, forearm site variation, right/left difference and time of the day on the skin surface $\mathrm{pH}$. Skin Res Technol. 2001;7(2):90-4. https://doi.org/10.1034/j.1600-0846.2001.70206.x.

28. Sempionatto JR, Lin M, Yin L, de la paz E, Pei K, Sonsa-ard T, et al. An epidermal patch for the simultaneous monitoring of haemodynamic and metabolic biomarkers. Nat Biomed Eng. 2021;5(7):737-48. https://doi.org/1 0.1038/s41551-021-00685-1.

29. Bowe WP, Joshi SS, Shalita AR. Diet and acne. J Am Acad Dermatol. 2010; 63(1):124-41. https://doi.org/10.1016/j.jaad.2009.07.043.

30. Pretorius RA, Bodinier M, Prescott SL, Palmer DJ. Maternal fiber dietary intakes during pregnancy and infant allergic disease. Nutrients. 2019;11(8). https://doi.org/10.3390/nu11081767.

31. Messina M, Messina V. The role of soy in vegetarian diets. Nutrients. 2010; 2(8):855-88. https://doi.org/10.3390/nu2080855.
32. Shang P, Shu Z, Wang Y, Li N, du S, Sun F, et al. Veganism does not reduce the risk of the metabolic syndrome in a Taiwanese cohort. Asia Pac J Clin Nutr. 2011;20(3):404-10.

33. Lin TJ, Tang SC, Liao PY, Dongoran RA, Yang JH, Liu CH. A comparison of Lcarnitine and several cardiovascular-related biomarkers between healthy vegetarians and omnivores. Nutrition. 2019;66:29-37. https://doi.org/10.101 6/j.nut.2019.03.019.

34. Lee MS, et al. A global overall dietary index: ODI-R revised to emphasize quality over quantity. Asia Pac J Clin Nutr. 2008;17(Suppl 1):82-6.

35. Lee MS, Huang YC, Su HH, Lee MZ, Wahlqvist ML. A simple food quality index predicts mortality in elderly Taiwanese. J Nutr Health Aging. 2011; 15(10):815-21. https://doi.org/10.1007/s12603-011-0081-x.

36. Chiu YF, Hsu CC, Chiu THT, Lee CY, Liu TT, Tsao CK, et al. Cross-sectional and longitudinal comparisons of metabolic profiles between vegetarian and non-vegetarian subjects: a matched cohort study. Br J Nutr. 2015;114(8): 1313-20. https://doi.org/10.1017/S0007114515002937.

37. Ryu JH, Seo YK, Boo YC, Chang MY, Kwak TJ, Koh JS. A quantitative evaluation method of skin texture affected by skin ageing using replica images of the cheek. Int J Cosmet Sci. 2014;36(3):247-52. https://doi.org/1 $0.1111 /$ ics.12120.

38. Ohshima H, Kinoshita S, Oyobikawa M, Futagawa M, Takiwaki H, Ishiko A, et al. Use of Cutometer area parameters in evaluating age-related changes in the skin elasticity of the cheek. Skin Res Technol. 2013;19(1):e238-42. https://doi.org/10.1111/j.1600-0846.2012.00634.x.

39. Ryu HS, Joo YH, Kim SO, Park KC, Youn SW. Influence of age and regional differences on skin elasticity as measured by the Cutometer. Skin Res Technol. 2008;14(3):354-8. https://doi.org/10.1111/j.1600-0846.2008.00302.x.

\section{Publisher's Note}

Springer Nature remains neutral with regard to jurisdictional claims in published maps and institutional affiliations.
Ready to submit your research? Choose BMC and benefit from:

- fast, convenient online submission

- thorough peer review by experienced researchers in your field

- rapid publication on acceptance

- support for research data, including large and complex data types

- gold Open Access which fosters wider collaboration and increased citations

- maximum visibility for your research: over 100M website views per year

At BMC, research is always in progress.

Learn more biomedcentral.com/submissions 